\title{
Assesment of colistin related side effects in premature neonates
}

\author{
Gülşah Kaya Aksoy ${ }^{1 \oplus}$, Sariye Elif Özyazıcı Özkan ${ }^{2 \oplus}$, Gönül Tezel ${ }^{2 \oplus}$, \\ Gülperi Timurtaş Dayar ${ }^{3 \oplus}$, Muhammet Köşker ${ }^{3 \oplus}$, Çağla Serpil Doğan ${ }^{1 \oplus}$ \\ Departments of ${ }^{1}$ Pediatric Nephrology, ${ }^{2}$ Neonatology and ${ }^{3}$ Pediatric Infectious Diseases, University of Health Sciences, Antalya \\ Training and Research Hospital, Antalya, Turkey.
}

\begin{abstract}
Background. Colistin is an antibiotic in the polymyxin group and is especially important in the elimination of multi-drug resistant gram negative bacteria. To date, there are many studies investigating colistin related side effects, especially nephrotoxicity. However, there are few studies involving premature neonates, and this study aimed to investigate the side effects of colistin in this particular patient group.
\end{abstract}

Methods. Between January 2016 and May 2019, the medical records of premature neonates treated with colistin were retrospectively reviewed. The diagnosis of acute kidney injury (AKI) was performed according to the modified neonatal KDIGO criteria. Serum electrolyte levels were recorded at the initiation of colistin treatment and 4-7 days after.

Results. A total of 47 premature neonates; with a median gestational age of 27 weeks and median weight of $970 \mathrm{~g}$ at birth were included in the study. The median postnatal day of colistin initiation was 24 days and mean duration of colistin therapy was $15.95 \pm 3.70$ days. Colistin was combined with aminoglycosides in $44.6 \%$ of the patients. Acute kidney injury was documented in $17.0 \%$ of premature neonates. ( $\mathrm{n}=6$ for stage $1, \mathrm{n}=2$ for stage 2 , none of the patients had stage 3). In univariate analysis, gestational age and concomitant aminoglycoside use were associated with AKI development $(\mathrm{OR}, 0.446 ; 95 \%$ CI 0.238-0.832; $\mathrm{p}=0.011$ and $\mathrm{OR}, 1.324 ; 95 \%$ CI $1.023-$ 7.584; $\mathrm{p}=0.024)$. Mean magnesium level significantly decreased after colistin treatment (1.70 \pm 0.84 vs. $1.57 \pm$ $0.29, \mathrm{p}=0.017)$ and the frequency of hypomagnesemia increased after colistin use $(78.7 \%$ vs. $91.5 \%, \mathrm{p}=0.031)$. Frequency of elevated AST increased from $23.4 \%$ to $44.7 \%$ following colistin use ( $p=0.031$ ).

Conclusions. Colistin-related side effects observed in premature neonates are not as common as in pediatric patients. Electrolyte imbalance is observed more frequently in this age group following colistin use. We suggest strict serum electrolyte level monitoring, especially magnesium, in premature neonates that are receiving colistin.

Key words: colistin, side effect, premature neonates, hypocalcemia, hypomagnesemia, acute kidney injury.

Nosocomial infections in neonatal intensive care units (NICUs) are among the most important factors that affect the survival of neonates. ${ }^{1}$ In particular, infections caused by multi-drug-resistant (MDR) bacteria have a more pronounced effect on mortality, and it is well known that these bacteria are becoming

\section{Gülşah Kaya Aksoy}

gkayaaksoy@gmail.com

Received 12th December 2019, revised 6th February 2020, accepted 24th February 2020. increasingly common in NICUs worldwide., ${ }^{2,3}$ In a study examining neonates with bacteremia, the frequency of MDR bacteremia was found to be $18.6 \%$ among all neonatal gram negative bacteremia cases. ${ }^{4}$

Colistin(colistimethate sodium) is a cyclopeptide antibiotic in the polymyxin group. ${ }^{5}$ Although many years have passed since its discovery, it is still being frequently investigated because of its potent effect on MDR gram negative bacteria. Colistin is especially recommended for the elimination of carbapenem-resistant isolates of 
Acinetobacter baumannii, Klebsiella pneumoniae and Pseudomonas aeruginosa. ${ }^{6}$ Colistin-related side effects were reported in the literature before the 1970s. ${ }^{7}$ In adult studies, the incidence of adverse events was close to $50 \%$, while the two most common side effects were nephrotoxicity and neurotoxicity. ${ }^{8}$

Colistin is attached to megaline in the proximal tubule because of its cationic structure, and accumulates in the proximal tubule with long-term use. ${ }^{9}$ Colistin is thought to increase cytoplasmic membrane permeability in proximal tubular cells, and cause an overflow of anion, cation and water into the cell and severely damaging them.,10 There are a number of studies evaluating colistin-related nephrotoxicity in the pediatric age group. ${ }^{11,12}$ However, such studies focusing on premature children are quite few. ${ }^{13}$

The aim of this study was to investigate the incidence of colistin-related side effects in premature neonates and to determine risk factors.

\section{Material and Methods}

\section{Study design and data collection}

Between January 2016 and May 2019, the medical records of premature neonates treated with colistin in the NICU of University of Health Sciences Antalya Training and Research Hospital were retrospectively reviewed using electronic health records and archived patient files. Prematurity was defined as birth before completing 37 weeks of gestation. Demographic characteristics, Apgar score at $5^{\text {th }}$ minute, comorbid conditions [low birth weight, respiratory support, umbilical/central venous catheter application, respiratory distress syndrome (RDS), patent ductus arteriosus (PDA), necrotizing enterocolitis (NEC), etc.] and all antimicrobial drugs used prior to colistin were recorded. Study exclusion criteria: a) neonates who received colistin treatment for less than 6 doses, b) those with kidney injury before starting colistin, c) neonates with congenital anomalies of the kidney and urinary system on ultrasonographic imaging. Permission for this study was officially granted by the Ethical Board of the University of Health Sciences, Antalya Training and Research Hospital [decision number: 194/May 20, 2019].

\section{Definitions}

Small for gestational age (SGA) was defined as birth weight $<10^{\text {th }}$ percentile for the gestational age. ${ }^{14}$ The diagnosis of PDA was confirmed by the presence of left-to-right shunt in echocardiographic examination in patients with PDA clinical symptoms. The diagnosis of NEC was made using Modified Bell's Staging Criteria. $^{15}$

The diagnosis of acute kidney injury (AKI) was performed according to the modified neonatal KDIGO criteria (stage 0: no change in serum creatinine; stage $1:>0.3 \mathrm{mg} / \mathrm{dl}$ increase in serum creatinine value in the last 48 hours or 150 to $200 \%$ increase in serum creatinine level compared to baseline; stage 2: an increase in serum creatinine level between 200 and 300\% compared to baseline; stage 3: an increase in serum creatinine level of $>300 \%$ compared to baseline, or a serum creatinine value $>2.5 \mathrm{mg} / \mathrm{dl}$ or renal replacement therapy). ${ }^{16}$

Serum creatinine, sodium, potassium, calcium, magnesium, alanine transaminase (ALT) and aspartate transaminase (AST) levels were recorded at the initiation of colistin treatment and 4 to 7 days after. Hyponatremia was defined as serum sodium levels $\leq 135 \mathrm{mmol} / \mathrm{L}$, hypokalemia $\leq 3.5 \mathrm{mmol} / \mathrm{L}$, hypocalcemia $\leq 8.5 \mathrm{mg} / \mathrm{dl}$, hypomagnesemia $\leq 1.9 \mathrm{mg} / \mathrm{dl}$, hypertransaminasemia ALT level $\geq 35 \mathrm{U} / \mathrm{L}$ and/ or AST level $\geq 50 \mathrm{U} / \mathrm{L}$.

\section{Colistin dosage}

Colistin was started either in neonates with MDR gram negative bacteremia in blood, urine or tracheal aspirate culture results or in neonates with clinical signs of sepsis despite the use of broad spectrum antibiotics. Multi-drugresistance was considered as the identification 
of a gram negative bacteria that was resistant to at least three different antibacterial groups. ${ }^{17}$ Colistin treatment was administered at $5 \mathrm{mg} /$ $\mathrm{kg}$ /day given in 3 doses dissolved in $5 \mathrm{ml}$ saline, for a minimum infusion duration of 30 minutes. The colistin formulation contained 150 mg of colistin base-equivalent colistimethate sodium (Colimycin; Kocak Farma, Istanbul, Turkey and Kolistate; Biem Farma, Ankara, Turkey). Postnatal age at the time of treatment with colistin and treatment duration were recorded. Colistin can be used as monotherapy or combined therapy with aminoglycoside or carbapenem in premature neonates.

\section{Statistical analysis}

Descriptive statistics were presented as frequency, percentage, mean, standard deviation (SD), median and interquartile range (IQR). The Shapiro-Wilk test, KolmogorovSmirnov test, histogram and Q-Q graphics were used for the evaluation of normality of distribution. The McNemar test was used in the analysis of relationships between categorical variables. For the comparison of continuous variables, the paired-samples t-test was used for variables that showed normal distribution, while the Wilcoxon test was used in those with non-normal distributions. Univariate logistic regression analyses were applied to identify risk factors for AKI. Odds ratios (ORs) were calculated with 95\% confidence intervals (CI). Statistical analyses were performed by using the SPSS version 21.0 package program for Windows (IBM, Armonk, NY). P values of $<0.05$ were accepted to show statistical significance.

\section{Results}

A total of 47 premature neonates (27 males, $57.4 \%$ ) were included in the study. Clinical features are presented in Table I. All patients required parenteral nutrition, and median parenteral nutrition duration was 27 days (range, 10-161 days; IQR, 23-37).

The median postnatal day of colistin initiation was 24 days (range, 9-74 days; IQR, 14-35) and mean duration of colistin therapy was $15.95 \pm$ 3.70 days. Culture positivity was present in 9 $(19.1 \%)$ of the neonates treated with colistin. In blood culture samples, Enterobacter cloacae was isolated in four, Acinetobacter baumannii and Klebsiella pneumoniae in one patient each, while in tracheal aspirate samples, Acinetobacter baumannii in one and Klebsiella pneumoniae in two patients. The antimicrobial drugs utilized before colistin treatment and combined with colistin are summarized in Table I.

The serum creatinine, serum electrolytes and transaminase levels evaluated before and during colistin treatment are presented in Table II. Acute kidney injury was documented in $17.0 \%$ of premature neonates. $(n=6$ for stage 1 , $\mathrm{n}=2$ for stage 2 , none of the patients had stage $3)$. The median gestational age was 25 weeks (range from 24-27 weeks) in neonates with AKI and 27 weeks (range from 23-34 weeks) in neonates without AKI ( $\mathrm{p}<0.001)$. Also, the median birth weight was $760 \mathrm{~g}$ (range from 650$1100 \mathrm{~g}$ ) in neonates with AKI and $1040 \mathrm{~g}$ (range from $610-2100 \mathrm{~g}$ ) in neonates without AKI ( $\mathrm{p}=$ 0.041). The incidence of AKI was higher among those in which aminoglycoside and colistin were combined compared to non-combination (23.8\% vs $11.5 \%$; $\mathrm{p}=0.031)$.

The risk factors for AKI in premature neonates were analyzed by univariate regression analysis. Gestational age and concomitant aminoglycoside use were associated with AKI development. On the other hand, Apgar score at 5th minute, birth weight, presence of PDA or NEC, and starting day of colistin were not significantly related with AKI (Table III).

Mean serum calcium and magnesium levels were significantly decreased after colistin treatment ( $9.59 \pm 0.84$ vs. $9.20 \pm 1.04, p=0.048$; $1.70 \pm 0.84$ vs. $1.57 \pm 0.29, p=0.017$, respectively). The number of patients with electrolyte imbalance before and during colistin treatment show that the frequency of hypomagnesemia and hypokalemia were increased after colistin use $(78.7 \%$ vs. $91.5 \%, \mathrm{p}=0.031 ; 4.3 \%$ vs. $27.7 \%$, $\mathrm{p}=0.007$, respectively) (Table II). 
Table I. Characteristics and outcomes of the study population $(n=47)$.

\begin{tabular}{lc}
\hline Variables & Values \\
\hline Sex (male), $\mathrm{n}(\%)$ & $27(57.4)$ \\
Median gestational age (weeks) & 27 (range 3-34; IQR 26-28) \\
Median birth weight (g) & 970 (range 610-2,600; IQR 780-1,100) \\
Low birth weight, $\mathrm{n}(\%)$ & $9(19.1)$ \\
Median 5th minute APGAR score & 41 (range 3-9; IQR 4-7) \\
Presence of umbilical catheter, $\mathrm{n}(\%)$ & $31(66.0)$ \\
Presence of central venous catheter, $\mathrm{n}(\%)$ & $43(91.5)$ \\
Need for respiratory support, $\mathrm{n}(\%)$ & \\
Comorbid conditions, $\mathrm{n}(\%)$ & $43(91.5)$ \\
$\quad$ Respiratory distress syndrome & $17(36.2)$ \\
Patent ductus arteriosus & $18(38.3)$ \\
$\quad$ Necrotizing enterocolitis & \\
Previous antibiotic exposure, $\mathrm{n}(\%)$ & $47(100.0)$ \\
Ampicillin & $35(74.5)$ \\
Aminoglycoside & $36(76.6)$ \\
Cephalosporin & $45(95.7)$ \\
Carbapenem & $35(78.7)$ \\
Amphotericin & $47(100)$ \\
Vancomycin/teicoplanin & \\
Concomitant antibiotics, $\mathrm{n}(\%)$ & $21(44.6)$ \\
Aminoglycoside & $3(6.4)$ \\
Cephalosporin & $38(80.8)$ \\
Carbapenem & $21(44.6)$ \\
Amphotericin & $39(83.0)$ \\
Vancomycin/teicoplanin & $46($ range $22-131 ; \mathrm{IQR} 45-105)$ \\
The median duration of hospitalization (days) & $4(8.5)$ \\
Mortality rate, $\mathrm{n}(\%)$ &
\end{tabular}

IQR: interquartile range

Median ALT and AST levels were significantly increased after colistin use (Table II). There was no change in the frequency of ALT elevation with the use of colistin. However, the rate of patients with AST elevation before colistin was $23.4 \%$, which increased to $44.7 \%$ with the use of colistin $(p=0.031)$.

\section{Discussion}

In our study, the incidence of AKI after colistin use was $17.0 \%$ in premature neonates. In a study evaluating extremely low birth weight prematures, the incidence of colistin-associated AKI was $14.3 \% .^{18}$ In a case-control study published by İpek et al. ${ }^{19}$, the frequency of nephrotoxicity was similar between prematures who had used colistin and those who had not ( $12.8 \%$ vs. $13.6 \%$, respectively). In the literature, the incidence of colistin-related nephrotoxicity observed in premature neonates was lower than that found in adult and pediatric studies. . $^{6,78,20}$ In a multicenter study from the United States, colistin-related nephrotoxicity was found to be 7 times higher in children $\geq 13$ years of age than in younger children. ${ }^{20}$

In ourstudy, gestational age was found to be a risk factor for colistin associated AKI in premature neonates. In a study evaluating 66 neonates by 
Table II. Changes in serum electrolytes and liver function tests after colistin use in premature neonates.

\begin{tabular}{lccc}
\hline Variables & Before colistin treatment & During colistin treatment & $\mathrm{p}$ \\
\hline Serum creatine (mg/dl) & $0.53 \pm 0.11$ & $0.61 \pm 0.26$ & $0.003^{*}$ \\
Serum sodium (mmol/L) & $138.31 \pm 4.39$ & $137.00 \pm 3.81$ & 0.055 \\
Frequency of hyponatremia (\%) & 23.4 & 34.0 & 0.359 \\
Serum potassium (mmol/L) & $4.56 \pm 0.56$ & $4.28 \pm 0.81$ & 0.068 \\
Frequency of hypokalemia (\%) & 4.3 & 27.7 & $0.007^{*}$ \\
Serum calcium (mg/dl) & $9.59 \pm 0.84$ & $9.20 \pm 1.04$ & 0.048 \\
Frequency of hypocalcemia (\%) & 8.5 & 12.8 & 0.754 \\
Serum magnesium (mg/dl) & $1.70 \pm 0.84$ & $1.57 \pm 0.29$ & $0.017^{*}$ \\
Frequency of hypomagnesemia (\%) & 78.7 & 91.5 & 0.031 \\
ALT (U/L), median (min.-max.) & $17(5-76)$ & $24(6-132)$ & $0.020^{*}$ \\
Frequency of high ALT level (\%) & 17.0 & 21.3 & 0.754 \\
AST (U/L), median (min.-max.) & $33(21-45)$ & $40(6-127)$ & $0.001^{*}$ \\
Frequency of high AST level (\%) & 23.4 & 44.7 & $0.031^{*}$ \\
\hline
\end{tabular}

*: $p<0.05$

ALT: alanine transaminase, AST: aspartate transaminase.

Table III. Evaluation of risk factors for acute kidney injury $(n=47)$.

\begin{tabular}{lccc}
\hline Risk factors & Odds ratio & $95 \% \mathrm{CI}$ & $\mathrm{p}$ \\
\hline Gestational age & 0.446 & $0.238-0.832$ & $0.011^{*}$ \\
Birth weight & 0.995 & $0.991-1.000$ & 0.061 \\
5th minute APGAR score & 0.742 & $0.467-1.180$ & 0.208 \\
PDA & 2.074 & $0.430-9.306$ & 0.377 \\
NEC & 0.479 & $0.086-2.683$ & 0.403 \\
Concomitant aminoglycoside use & 0.258 & $0.053-1.264$ & 0.095 \\
Day colistin started & 0.945 & $0.875-1.021$ & 0.154 \\
\hline
\end{tabular}

PDA: patent ductus arteriosus, NEC: necrotizing enterocolitis OR: odds ratio, CI: confidence interval.

Ilhan et al. ${ }^{18}$, colistin associated AKI incidence was $14.3 \%$ in neonates with birth weight $<1,500$ $\mathrm{g}$ and $2.6 \%$ in those with birth weight $>1500$ $\mathrm{g}$; but this difference was not statistically significant $(\mathrm{p}=0.15)$. In this study, we also found that colistin and aminoglycoside combination was associated with AKI development in premature neonates. The association between colistin-related nephrotoxicity and concomitant aminoglycoside use has been particularly emphasized in adult studies. ${ }^{21}$

In the current study, electrolyte imbalance developed in most of the premature neonates who had used colistin. With the use of colistin, both mean serum magnesium concentration decreased and the frequency of neonates with hypomagnesemia also increased. In accordance with our study, most of the previously published studies evaluating neonates reported low serum magnesium levels. ${ }^{13,19,20,22}$ In the study by Alan et al. $^{13}$, serum magnesium concentration decreased significantly after the use of colistin; to such a degree that in $52 \%$ of patients, magnesium replacement was required. Since the change in serum magnesium concentration was observed especially in premature neonates, this condition was thought to be due to colistinrelated nephrotoxicity, and the immature structure and lower number of nephrons. ${ }^{18}$

In our study, not only hypomagnesemia but also calcium and potassium imbalance were observed in premature neonates. In a study 
evaluating 21 preterm neonates after colistin use, potassium and calcium replacement were required in $52 \%$ and $33 \%$ of patients, respectively. ${ }^{13}$ Among premature neonates, low birth weight patients were found to have higher risk for potassium and calcium imbalance. ${ }^{18}$ In the literature, a case mimicking tubular disorders after colistin use has been reported. A newborn with a gestational age of 28 weeks was presented with a Bartter-like syndrome developed after colistin use, and also metabolic alkalosis. ${ }^{23}$

In our study, we found a significant increase in both transaminase levels, especially AST, with the use of colistin. To our knowledge, hepatotoxicity has not been reported among the side effects of colistin. However, despite this finding, it is highly likely that this increase in transaminase levels is due to other drugs, clinical characteristics, sepsis, and multiple organ dysfunction syndrome.

The most important limitation of this study is its retrospective nature. Secondly, in this study, serum creatinine was used as a marker of renal damage. It has been shown that serum creatinine level does not increase unless $25-50 \%$ of renal functions are impaired in neonates. ${ }^{24}$ Cumulative doses and drug interactions of all antimicrobial drugs used prior to colistin could not be evaluated within the scope of the study. Because of the prolonged renal effects of some antimicrobial drugs (amphotericin B, aminoglycoside, etc.) used just before the colistin, we may have been evaluated as the side effects of colistin. Finally, as the urine electrolyte levels were not evaluated, we thought that electrolyte imbalance after colistin use may be due to renal tubular loss. However, despite these limitations, we think that it will contribute to the literature as it investigates the side effects related to colistin use in neonates with low gestational age.

The current data suggests that the frequency of colistin associated AKI in premature neonates is lower than children and adults. It also seems that electrolyte imbalance is observed more frequently in this age group following colistin use; however, it is also possible that this change is associated with various other parameters. Nevertheless, we suggest strict serum electrolyte level monitoring, especially magnesium, in premature neonates that are receiving colistin.

\section{REFERENCES}

1. Stoll BJ, Hansen N, Fanaroff AA, et al. Lateonset sepsis in very low birth weight neonates: the experience of the NICHD Neonatal Research Network. Pediatrics 2002; 110 (2 Pt 1): 285-291.

2. Oliveira PMN, Buonora SN, Souza CLP, et al. Surveillance of multidrug-resistant bacteria in pediatric and neonatal intensive care units in Rio de Janeiro State, Brazil. Rev Soc Bras Med Trop 2019; 52: e20190205.

3. Yusef D, Shalakhti T, Awad S, Algharaibeh $\mathrm{H}$, Khasawneh W. Clinical characteristics and epidemiology of sepsis in the neonatal intensive care unit in the era of multi-drug resistant organisms: a retrospective review. Pediatr Neonatol 2018; 59: 3541.

4. Tsai MH, Chu SM, Hsu JF, et al. Risk factors and outcomes for multidrug-resistant gram-negative bacteremia in the NICU. Pediatrics 2014; 133: e322-e329.

5. Grégoire N, Aranzana-Climent V, Magréault S, Marchand S, Couet W. Clinical pharmacokinetics and pharmacodynamics of colistin. Clin Pharmacokinet 2017; 56: 1441-1460.

6. Antachopoulos C, Iosifidis E. Colistin use in neonates and children with infections due to carbapenemresistant bacteria. Pediatr Infect Dis J 2017; 36: 905907.

7. Hoeprich PD. The polymyxins. Med Clin North Am 1970; 54: 1257-1265.

8. Falagas ME, Kasiakou SK. Toxicity of polymyxins: a systematic review of the evidence from old and recent studies. Crit Care 2006; 10: R27.

9. Suzuki T, Yamaguchi H, Ogura J, Kobayashi M, Yamada T, Iseki K. Megalin contributes to kidney accumulation and nephrotoxicity of colistin. Antimicrob Agents Chemother 2013; 57: 6319-6324.

10. Sahbudak Bal Z, Kamit Can F, Yazici P, et al. The evaluation of safety and efficacy of colistin use in pediatric intensive care unit: results from two reference hospitals and review of literature. J Infect Chemother 2018; 24: 370-375.

11. Jajoo M, Kumar V, Jain M, Kumari S, Manchanda V. Intravenous colistin administration in neonates. Pediatr Infect Dis J 2011; 30: 218-221. 
12. Kumar PP, Giri SR, Shaikh FAR, Panigrahy N, Chirla D. Safety and efficacy of intravenous colistin in children. Indian Pediatr 2015; 52: 129-130.

13. Alan S, Yildiz D, Erdeve O, et al. Efficacy and safety of intravenous colistin in preterm infants with nosocomial sepsis caused by Acinetobacter baumannii. Am J Perinatol 2014; 31: 1079-1086.

14. Kramer MS. Determinants of low birth weight: methodological assessment and meta-analysis. Bull World Health Organ 1987; 65: 663-737.

15. Walsh MC, Kliegman RM. Necrotizing enterocolitis: treatment based on staging criteria. Pediatr Clin North Am 1986; 33: 179-201.

16. Selewski DT, Charlton JR, Jetton JG, et al. Neonatal acute kidney injury. Pediatrics 2015; 136: e463-e473.

17. Magiorakos AP, Srinivasan A, Carey RB, et al. Multidrug-resistant, extensively drug-resistant and pandrug-resistant bacteria: an international expert proposal for interim standard definitions for acquired resistance. Clin Microbiol Infect 2012; 18: 268-281.

18. Ilhan O, Bor M, Ozdemir SA, Akbay S, Ozer EA. Efficacy and safety of intravenous colistin in very low birth weight preterm infants. Paediatr Drugs 2018; 20: 475-481.
19. İpek MS, Aktar F, Okur N, Celik M, Ozbek E Colistin use in critically ill neonates: a case-control study. Pediatr Neonatol 2017; 58: 490-496.

20. Tamma PD, Newland JG, Pannaraj PS, et al. The use of intravenous colistin among children in the United States: results from a multicenter, case series. Pediatr Infect Dis J 2013; 32: 17-22.

21. Temocin F, Erdinc S, Tulek N, Demirelli M, Bulut C, Ertem G. Incidence and risk factors for colistinassociated nephrotoxicity. Jpn J Infect Dis 2015; 68: 318-320.

22. Al-Lawama M, Aljbour H, Tanash A, Badran E. Intravenous colistin in the treatment of multidrugresistant Acinetobacter in neonates. Ann Clin Microbiol Antimicrob 2016; 15: 8.

23. Cakir U, Alan S, Zeybek C, et al. Acquired Bartterlike syndrome associated with colistin use in a preterm infant. Ren Fail 2013; 35: 411-413.

24. Jetton JG, Askenazi DJ. Acute kidney injury in the neonate. Clin Perinatol 2014; 41: 487-502. 